Published by Al-Nahrain College of Medicine P-ISSN 1681-6579

E-ISSN 2224-4719

Email: iraqijms@colmed-alnahrain.edu.iq

http://www.colmed-alnahrain.edu.iq

http://www.iraqijms.net

Iraqi JMS 2019; Vol. 17(2)

\title{
Genomic Biomarkers in Endometrial Carcinoma
}

\author{
Noora M. Kareem CABMS (Anatomic Pathology) \\ Dept. of Pathology and Forensic Medicine, College of Medicine, Al-Nahrain University, Baghdad, Iraq
}

\begin{abstract}
Endometrial carcinoma is the second most common gynecological cancer in developing countries after cervical carcinoma and its incidence is increasing due to the rise in the rate of obesity. Diagnosis depend on invasive test (biopsy) with no routine screening investigation available for either general population or high-risk group, there are several types of biomarkers that can be used for diagnosis, prognosis and management but none are available for routine clinical practice. Following the discovery of the new gene-based classifications of endometrial cancer, the use of these gene-based biomarkers will be the cornerstone in the early diagnosis and management for endometrial carcinoma patients in the coming years.
\end{abstract}

Keywords Endometrial carcinoma, screening, PTEN, miRNA, P53, circulating tumor DNA, genomic classification Citation Kareem NM. Genomic biomarkers in endometrial carcinoma. Iraqi JMS. 2019; 17(2): 100-102. doi: 10.22578/IJMS.17.2.1

List of abbreviation: ctDNA = Circulating tumor DNA, POLE $E D M=$ Polymerase $E$ mutated, $M M R-D=$ Mismatch repair deficient, PTEN $=$ The phosphatase and tensin homolog,

$\mathrm{E}$ ndometrial carcinoma represents the most common gynecologic malignancy in developed countries and the second most common gynecologic malignancy in developing countries after cervical cancer $(1,2)$. It is anticipated that the incidence of uterine cancer will increase to a higher rate worldwide in the following years due to the increasing rate of obesity. Most patients present in the post-menopausal years of age, the peak incidence at $70-74$ years. The principal clinical presentation of endometrial carcinoma is postmenopausal bleeding. $81-83 \%$ of patients will be discovered at stages I-II (3). The 5 years survival rates decrease dramatically from Stages I (95\%) reaching down to $14 \%$ in stage IV (4). Histologically there are two major categories of endometrial carcinoma; each has a different set of risk factors: type I (endometrioid) and type II (non-endometrioid, e.g., serous, squamous, clear cell, undifferentiated and carcinosarcoma). The endometroid types, which represent about $85 \%$ of the cases are typically estrogen dependent and low grade. Nevertheless, grade three tumors are more aggressive with overlapping clinical features with Type II endometrial carcinomas ${ }^{(5)}$.

\section{Screening}

Currently there is no routine screening for the general population for endometrial carcinoma. Since Lynch syndrome carries a high risk of developing endometrial cancer reaching to $60 \%$ lifetime risk, regular follow up with ultrasound and endometrial biopsy are offered to Lynch syndrome female patients and their first-degree relatives starting from 35 years of age, but these measures have not been shown to result in an earlier finding and diagnosis of endometrial carcinoma ${ }^{(6)}$. 


\section{Biomarkers}

Biomarker can be defined according to The National Cancer Institute as 'a biological molecule found in blood, other bodily fluids, or tissues that is a sign of a normal or abnormal process, or of a condition or disease' (7), the biomarkers can be used for diagnosis, prognosis, screening or treatment monitoring. many types of biomarkers had been researched focusing on predicting the probability of emergence of endometrial carcinoma from endometrial hyperplasia but none of them are available in practice ${ }^{(8)}$.

\section{Genomic biomarkers}

\section{P53}

P53 is a tumor suppressor gene, which can act as a trigger to cellular responses that can lead to cell-cycle arrest, differentiation, apoptosis, senescence, inhibition of angiogenesis, and DNA repair (9). Many studies investigate the role of p53 in both endometrial hyperplasia and endometrial carcinoma like D'Andrilli et al. (10) who found that p53 gene mutation is present in the aggressive variant of endometrial carcinoma and undetectable in the hyperplastic endometrium.

Mirakhor Samani et al. (11) who had investigated the expression of p53 in endometrial hyperplasia, endometrial carcinoma and normal endometrium and concluded that p53 overexpression is found in endometrial carcinoma and can be used for risk stratification and screening purposes.

\section{PTEN}

The phosphatase and tensin homolog (PTEN) is a tumor suppressor gene that plays a vital role in preserving the chromosomal stability (12). Mutation of PTEN is the most common early genetic change in type I endometrial carcinoma, found in $83 \%$ of cases ${ }^{(13)}$. Abd ElMaqsoud et al. ${ }^{(14)}$ had suggested that PTEN expression has a role in early stages of endometrial carcinoma, however, a recent study done by Raffone et al. ${ }^{(15)}$ showed that PTEN expression has a low diagnostic usefulness in differentiating between endometrial hyperplasia and endometrial carcinoma and its use should be reconsidered.

\section{Genomic classification and next-generation sequencing}

Recently, The Proactive Molecular Risk Classifier for Endometrial Cancer had classified endometrial cancers into four genomic subtypes: polymerase E mutated (POLE EDM), mismatch repair deficient (MMR-D); p53 wild type and p53 abnormal. POLE EDMs had a favorable prognosis and tends to occur in thin and young women. MMR-D tumors were similar to POLE pathologically but with a worse outcome, this subtype may be related to Lynch syndrome so genetic test is mandatory in this category. The highest percentage of high grade, non-endometroid tumors were in the p53 abnormal category with the worst prognosis (16). These genomic and clinical classifiers may assist in risk-Stratify patients for chemotherapy or radiotherapy treatment and come up with a personalized follow up plans for each patient ${ }^{(17)}$.

\section{MicroRNAs}

Small non-coding RNAs are contributing in different transcriptional processes, including carcinogenesis, and can be found in several body fluids ${ }^{(18)}$, some miRNAs can be used to distinguish between early and advanced endometrial carcinoma ${ }^{(19)}$. 18 urine cell-free miRNAs were investigated in a pilot prospective study of endometrial and ovarian cancers and showed a prominent suppression of MiR-106b in endometrial cancer ${ }^{(18)}$. These urine microRNAs can be considered as potential biomarkers in gynaecological cancers.

\section{Circulating tumor DNA}

Circulating tumor DNA (ctDNA) has been documented as a useful biomarker in early diagnosis of cancer. They can detect both genetic and epigenetic mutations. They are observable in plasma, eliminated by the kidneys and so they can be detected in urine. ctDNA had been extensively studied in different cancers such as, prostate, breast, lung and colorectal. Regarding endometrial 
carcinoma there are some continuous studies, the result of which are awaited (20).

For the time being, there is no biomarker that can be used routinely in endometrial cancer diagnosis and prognosis assessment. Molecular biomarkers cannot be detected without tissue, which is obtained by surgical procedures causing morbidity and mortality. After the emergence of new genomic classification of endometrial carcinoma further studies is needed for a better management of endometrial cancer patients.

\section{References}

1. Jemal A, Bray F, Center MM, et al. Global cancer statistics. CA: A Cancer Journal for Clinicians, 2011; 61(2), 69-90. doi:10.3322/caac.20107.

2. American Cancer Society. Global cancer facts \& figure. $3^{\text {rd }}$ ed. Atlanta, GA, USA, 2012.

3. Cancer Research UK. Uterine cancer incidence statistics. $2014 . \quad$ URL: https://www.cancerresearchuk. org/healthprofessional/cancer-statistics/statistics-by-cancertype/ uterine-cancer/incidence\#heading-One

4. Office for National Statistics. Cancer survival in England: national estimates for patients followed up to $2017 . \quad$ URL: https://www.ons.gov.uk/peoplepopulationandcom munity/healthandsocialcare/conditionsanddiseases /bulletins/cancersurvivalinengland/nationalestimat esforpatientsfollowedupto2017.

5. Buhtoiarova TN, Brenner CA, Singh M. Endometrial carcinoma: role of current and emerging biomarkers in resolving persistent clinical dilemmas. Am J Clin Pathol. 2016; 145(1): 8-21. doi: 10.1093/ajcp/aqv014.

6. British Gynecological Cancer Society. BGCS Uterine Cancer Guidelines: Recommendations for practice. 2014.

URL: https://bgcs.org.uk/BGCS\%20Endometrial\%20Guid elines\%202017. Pdf.

7. National Cancer Institute Dictionary of Cancer Terms. $2018 . \quad$ URL: https://www.cancer.gov/publications/dictionaries/ cancer-terms/def/biomarker.

8. Hutt $S$, Tailor A, Ellis, et al. The role of biomarkers in endometrial cancer and hyperplasia: a literature review. Acta Oncol. 2019; 58(3): 342-52. doi: 10.1080/0284186X.2018.1540886.

9. Giono LE, Manfredi JJ. The p53 Tumor suppressor participates in multiple cell cycle checkpoints. J Cell
Physiol. 2006; 209(1): 13-20. doi: 10.1002/jcp.20689.

10. D’Andrilli G, Bovicelli A, Paggi MG, et al. New insights in endometrial carcinogenesis. J Cell Physiol. 2012; 227(7): 2842-6. doi: 10.1002/jcp.24016.

11. Mirakhor Samani S, Ezazi Bojnordi T, Zarghampour $M$, et al. Expression of p53, Bcl-2 and Bax in endometrial carcinoma, endometrial hyperplasia and normal endometrium: a histopathological study. J Obstet Gynaecol. 2018; 38(7): 999-1004. doi: 10.1080/01443615.2018.1437717.

12. Yin $Y$, Shen WH. PTEN: a new guardian of the genome. Oncogene. 2008; 27(41): 5443-53. doi: 10.1038/onc.2008.241.

13. Boruban MC, Altundag K, Kilic GS, et al. From endometrial hyperplasia to endometrial cancer: insight into the biology and possible medical preventive measures. Eur J Cancer Prev. 2008; 17(2): 133-8. doi: 10.1097/CEJ.0b013e32811080ce.

14. Abd El-Maqsoud NM, El-Gelany S. Differential expression patterns of PTEN in cyclic, hyperplastic and malignant endometrium: its relation with ER, PR and clinicopathological parameters. J Egypt Nat Cancer Inst. 2009; 21(4): 323-1.

15. Raffone A, Travaglino A, Saccone G, et al. Loss of PTEN expression as diagnostic marker of endometrial precancer: A systematic review and meta-analysis. Acta Obstet Gynecol Scand. 2019; 98(3): 275-86. doi: 10.1111/aogs.13513.

16. Talhouk A, McConechy MK, Leung $S$, et al. Confirmation of ProMisE: A simple, genomics-based clinical classifier for endometrial cancer. Cancer. 2017; 123(5): 802-13. doi: 10.1002/cncr.30496.

17. Piulats JM, Matias-Guiu X. Immunotherapy in endometrial cancer: in the nick of time. doi: 10.1158/1078-0432.CCR-16-1820.

18. Záveský L, Jandáková $E$, Turyna $R$, et al. Evaluation of cell-free urine micrornas expression for the use in diagnosis of ovarian and endometrial cancers. A pilot study. Pathol Oncol Res. 2015; 21(4): 1027-35. doi: 10.1007/s12253-015-9914-y.

19. Jurcevic $S$, Klinga-Levan $K 1$, Olsson $B$, et al. MicroRNA expression in human endometrial adenocarcinoma. BMC Cancer. 2016; 16: 261. doi: 10.1186/s12885-016-2296-z.

20. Han X, Wang J, Sun Y. Circulating tumor DNA as biomarkers for cancer detection. Genomics Proteomics Bioinformatics. 2017; 15(2): 59-72. doi: 10.1016/j.gpb.2016.12.004.

E-mail: dr.nooramk@gmail.com Dr.nooramkar@colmed-alnahrain.edu.iq 